\title{
ON THE FINITE STRIP PROBLEM
}

\section{BY CARL E. PEARSON (Harvard University)}

1. Introduction. Many two-dimensional problems of physical interest reduce to the problem of solving a singular integral equation, with finite limits, of the form

$$
\int_{a}^{b}[P(x-t) \ln |x-t|+Q(x-t)] f(t) d t=g(x), \quad a<x<b,
$$

where $P, Q, g$ are prescribed well-behaved functions. For example, let a perfect fluid move with constant velocity parallel to the $x$-axis. If the portion $(a, b)$ of the $x$-axis is occupied by a thin metal strip heated to a prescribed temperature $g(x)$, then the temperature distribution in the fluid is easily obtained if the heat-source intensity $f(t)$ in the interval $(a, b)$ is known. This quantity $f(t)$ satisfies an integral equation of the form of Eq. (1) where the kernel (quantity in square brackets) is now proportional to [exp $\alpha(x-t) K_{0}(\alpha|x-t|)$ ]; here $K_{0}$ is the modified Hankel function and $\alpha$ is a constant involving the properties of the fluid.

No general closed-form expressions for the solution of Eq. (1) are known. In practice, three different techniques of approximation are used, depending on the length of the interval $(a, b)$. For intervals $(a, b)$ so long that the strip may be considered semi-infinite, the solution $f(t)$ near each end of the strip may be obtained by the Wiener-Hopf method [1] and these two end solutions may be superposed to give a practically useful solution for the entire strip. If on the other hand the interval is so short that only the zeroth order term of $P(x-t)$ need be considered, use can be made of Carleman's formula [2] for the solution of

$$
\int_{-1}^{1} f(t) \ln |x-t| d t=g(x)
$$

which is

$f(x)=\frac{1}{\pi^{2}\left(1-x^{2}\right)^{1 / 2}} \int_{-1}^{1} \frac{g^{\prime}(s) \cdot\left(1-s^{2}\right)^{1 / 2} d s}{s-x}-\frac{1}{\pi^{2}\left(1-x^{2}\right)^{1 / 2} \ln 2} \int_{-1}^{1} \frac{g(s) d s}{\left(1-s^{2}\right)^{1 / 2}}$.

Here and in future integrals of the form of the first term of Eq. (3) are to be interpreted in the principal value sense.

The in-between case of an interval of moderate length is always troublesome and usually requires either infinite series expansions or numerical techniques. In the following, a method will be described which gives an exact solution (in closed form involving simple integrals) of Eq. (1) for that case in which $P$ and $Q$ are $n$th order polynomials. The method involves the solution of a set of $n$ linear equations* in $n$ unknowns and so becomes tedious as $n$ increases; nevertheless, its advantages of exactness and straightforwardness of calculation should make it useful in certain "in-between" finite strip problems.

Before proceeding, some general comments concerning Eq. (1) may be of interest. Firstly, if all terms except $\int f(t) 1 n|x-t| d t$ are placed on the right hand side and

Received April 25, 1956.

*The coefficients of these linear equations are expressible in terms of Bessel functions at worst, which are easily obtainable from tables; thus no numerical integration is necessary. 
temporarily incorporated into $g(x)$, then the use of Eq. (3) with this modified $g(x)$ results in a non-singular Fredholm integral equation for $f(x)$. This method (due to Carleman [3]) is of theoretical value but the resulting Fredholm determinants are too complicated for practical use. Secondly, attempts to extend the Wiener-Hopf method to equations of the form (1) have often been made; although the various Fourier transforms involved can be determined within entire functions, the essential difficulty seems to lie in meeting the requirement that an inverse transform vanish over selected portions of the real axis-a condition which is automatically met in the Wiener-Hopf method but not here. Thirdly, an interesting approach suggested by Latta [4] utilizes the differential equation satisfied by the kernel function to obtain a differential equation for the solution in those cases where $g(x)$ is a simple function (such as a constant or power of $x$ ). The differential equation may then be solved by series expansions.

2. Solution of Eq. (1) for polynomial $P$ and $Q$. For convenience, the limits of integration will be chosen as -1 and +1 . Then define, for any complex point $z$ not in the strip $(-1,1)$, the analytic function $S(z)$ by

$$
S(z)=\left(z^{2}-1\right)^{1 / 2} \int_{-1}^{1}\left[P(z-t) \ln \left(\frac{z-t}{z+1}\right)+Q(z-t)\right] f(t) d t,
$$

where $\left(z^{2}-1\right)^{t}$ has the branch line $(-1,1)$ and behaves like $+z$ for large $z$, and where

$$
\ln \left(\frac{z-t}{z+1}\right)=\ln (z-t)-\ln (z+1) \text {. }
$$

Here and in future the value of $\ln (z+x)$, real $x$, is chosen as real for $z$ on the real axis to the right of the point $-x$; the branch line runs from $-x$ to $-\infty$.

Clearly, $S(z)$ is single-valued outside the strip. Also, the limiting values of $S(z)$ exist as $z$ approaches a point $x$ on the interval $(-1,1)$ from above or below, and are given respectively by

$$
\begin{aligned}
& S(x+)=i\left(1-x^{2}\right)^{1 / 2}\left[g(x)+\pi i \int_{x}^{1} P(x-t) f(t) d t\right.\left.-\left(\int_{-1}^{1} P(x-t) f(t) d t\right) \ln (1+x)\right], \\
& S(x-)=-i\left(1-x^{2}\right)^{1 / 2}\left[g(x)-\pi i \int_{x}^{1} P(x-t) f(t) d t\right. \\
&\left.-\left(\int_{-1}^{1} P(x-t) f(t) d t\right) \ln (1+x)\right],
\end{aligned}
$$

where roots and logarithms of real positive quantities are to be interpreted in the conventional principal-value manner. It follows that

$$
S(x+)-S(x-)=2 i\left(1-x^{2}\right)^{1 / 2}\left[g(x)-\left(\int_{-1}^{1} P(x-t) f(t) d t\right) \ln (1+x)\right]
$$

and therefore, by a property of Cauchy integrals [5],

$$
S(z)=\frac{1}{\pi} \int_{-1}^{1}(s-z)^{-1}\left(1-s^{2}\right)^{1 / 2}\left[g(s)-\left(\int_{-1}^{1} P(s-t) f(t) d t\right) \ln (1+s)\right] d s+T(z),(6)
$$


where $T(z)$ is entire. [ $T(z)$ could conceivably have isolated singularities at \pm 1 ; however, the order of magnitude of the other terms in Eq. (6)-assuming that $f(t)$ has no worse than a root singularity at these points - is such as to grow too slowly in the neighborhood of \pm 1 , so that no isolated singularity can exist.] Because the integral term in Eq. (6) tends to zero as $z$ approaches infinity, $T(z)$ must be the "entire part" of $S(z)$-i.e., the positive power portion of the Laurent expansion of $S(z)$ for the region outside the unit circle. Such a positive-power portion of a Laurent expansion can always be exhibited as a contour integral by means of Cauchy's integral theorem, or alternatively as a residue at infinity; however, it is most convenient for our present purposes to use Eq. (4) directly.

Consider next the quantity $S(x+)+S(x-)$. Using Eq. (5) and again a property of Cauchy integrals [5] it is easily seen that

where

$$
\int_{x}^{1} P(x-t) f(t) d t=h(x)
$$

$$
\begin{aligned}
h(x)=-\left(1-x^{2}\right)^{-1 / 2}\left\{\frac{1}{\pi^{2}} \int_{-1}^{1}(s\right. & -x)^{-1}\left(1-s^{2}\right)^{1 / 2}[g(s) \\
& \left.\left.-\left(\int_{-1}^{1} P(s-t) f(t) d t\right) \ln (1+s)\right] d s+\frac{1}{\pi} T(x)\right\} .
\end{aligned}
$$

If now Eqs. (4), (7), and (8) are examined, it will be seen that although the unknown $f(x)$ occurs on the right-hand side of Eq. (7), it does so only in the form of the $(n+2)$ constants

$$
c_{r}=\int_{-1}^{1} t^{r} f(t) d t, \quad r=0,1,2, \cdots, n+1,
$$

where $n$ is the greater of the orders of the polynomials $P$ and $Q$. Consequently, when Eq. (7) is solved for $f(x)$ (see Sec. 3 ) it is only necessary to multiply the result by various powers of $x$ and integrate between -1 and +1 in order to find linear equations relating these constants. Two such linear equations can always be obtained directly from Eq. (7) by multiplying both sides by $\left(1-x^{2}\right)^{\frac{1}{2}}$ and setting $x=+1$ or -1 . It may be verified that, with the exception of the integrals involving $g(s)$, all integrals which occur in the process of setting up these various linear equations are easily expressed in terms of tabulated functions (in fact, nothing worse than Bessel functions). In this connection, the integrals tabulated in Sec. 5 are of value.

In passing, an interesting alternative method of evaluating the $c_{r}$ may be mentioned. Suppose that the solution $m_{r}(x)$ were known for the equation

$$
\int_{-1}^{1}[P(t-x) \ln |t-x|+Q(t-x)] f_{1}(t) d t=x^{r} .
$$

If Eq. (1) is then multiplied by $m_{r}(x)$ and integrated with respect to $x$ from -1 to +1 , the result is

$$
c_{r}=\int_{-1}^{1} t^{r} f(t) d t=\int_{-1}^{1} m_{r}(x) g(x) d x .
$$

3. Solution of Eq. (7). Taking Laplace transforms of Eq. (7) and inverting gives

$$
f(x)=\frac{d}{d x} \int_{x}^{1} M(t-x) h^{\prime}(t) d t
$$


where $M(x)$ is the inverse transform of $\left[s^{2} p_{1}(s)\right]^{-1}, p_{1}(s)$ being the transform of $P(-x)$.

In Eq. (8), $h(x)$ has the apparent factor $\left(1-x^{2}\right)^{-\frac{1}{3}}$ which would make for integration difficulties in Eq. (9), where $h^{\prime}$ occurs. However, Eq. (7) shows that $h(1)=0$ and $h(-1)=$ finite (provided $f(x)$ is integrable) and consequently the factor $\left(1-x^{2}\right)^{-\frac{1}{3}}$ must be cancelled by the remaining terms. This cancellation could be carried out explicitly if desired, by multiplying both sides of Eq. (7) by $\left(1-x^{2}\right)^{1}$ and setting $x=+1$ and -1 ; if the first of these equations is multiplied by $\left(\frac{1}{2}\right)(1+x)\left(1-x^{2}\right)^{-1}$ and the second by $\left(\frac{1}{2}\right)(1-x)\left(1-x^{2}\right)^{-\frac{1}{1}}$ and if the two resulting equations are added to Eq. (8), the factor $\left(1-x^{2}\right)^{-\frac{1}{2}}$ will be found to cancel.* It will in fact be found that as $x \rightarrow 1, h(x)$ vanishes like $(1-x)^{4}$; this is easily seen to imply that $f(x)$ behaves like $(1-x)^{-\frac{1}{4}}$ as $x \rightarrow 1$, and therefore similarly as $x \rightarrow-1$.

4. First example. Set $P=1, Q=0$ [see Eq. (2)]. Then a consideration of the entire part of Eq. (4) shows that $T(x)$ is simply a constant:

$$
T(x)=-\int_{-1}^{1} f(t) d t-\int_{-1}^{1} t f(t) d t
$$

These two constants, $c_{0}$ and $c_{1}$, may be found by multiplying both sides of Eq. (7) by $\left(1-x^{2}\right)^{\frac{1}{3}}$ and setting $x=+1$ and -1 . The results are

$$
\begin{gathered}
: 0=-\frac{1}{\pi \ln 2} \int_{-1}^{1} \frac{g(s) d s}{\left(1-s^{2}\right)^{1 / 2}}, \\
c_{1}=-\frac{1}{\pi} \int_{-1}^{1} \frac{s g(s) d s}{\left(1-s^{2}\right)^{1 / 2}} .
\end{gathered}
$$

Then using Eq. (9) gives

$$
\begin{aligned}
f(x)=\frac{d}{d x}\left[( 1 - x ^ { 2 } ) ^ { - 1 / 2 } \left\{\frac{1}{\pi^{2}} \int_{-1}^{1} \frac{\left(1-s^{2}\right)^{1 / 2} g(s)}{s-x} d s\right.\right. \\
\left.\left.\quad-\frac{c_{0}}{\pi^{2}} \int_{-1}^{1} \frac{\left(1-s^{2}\right)^{1 / 2} \ln (1+s)}{s-x} d s-\frac{1}{\pi}\left(c_{0}+c_{1}\right)\right\}\right] .
\end{aligned}
$$

From the fact that Eqs. (3) and (10) must be identical, the identity (11) can be deduced.

5. Useful integrals. In the application of this method, a number of definite integrals continually occur; for convenience, the important ones are collected here. The first is derived as just mentioned, and the others by straightfoward contour integration.

$$
\begin{aligned}
& \int_{-1}^{1} \frac{\left(1-s^{2}\right)^{1 / 2} \ln (1+s) d s}{s-x}=\pi\left[x \ln 2-1+\left(1-x^{2}\right)^{1 / 2}\left(\frac{\pi}{2}-\sin ^{-1} x\right)\right] \\
& \int_{-1}^{1}(1+s)^{m}(1-s)^{r} \ln (1+s) d s \\
& =2^{m+r+1} \frac{\Gamma(m+1) \Gamma(r+1)}{\Gamma(m+r+2)}\{\ln 2+\psi(m+1)-\psi(m+r+2)\},
\end{aligned}
$$

*In carrying out the details of this discussion, it is advantageous to use an alternative form for $T(x)$ found by use of a contour integral as previously mentioned, with the contour shrunk to envelop the strip $(-1,1)$. 
where because of the present rationality of $m, r$, the psi-functions are expressible in terms of elementary functions. For example,

$$
\begin{gathered}
\int_{-1}^{1}\left(\frac{1+s}{1-s}\right)^{1 / 2} \ln (1+s) d s=\pi(1-\ln 2), \\
\int_{-1}^{1}\left(\frac{1-s}{1+s}\right)^{1 / 2} \ln (1+s) d s=-\pi(1+\ln 2), \\
\int_{-1}^{1} \frac{s F(s) d s}{s-x}=\int_{-1}^{1} F(s) d s+x \int_{-1}^{1} \frac{F(s) d s}{s-x}, \\
\int_{-1}^{1} \frac{\left(1-s^{2}\right)^{1 / 2} d s}{s-x}=-\pi x, \quad \int_{-1}^{1} \frac{\left(1-s^{2}\right)^{-1 / 2} d s}{s-x}=0, \\
\int_{-1}^{1} \frac{(1+s)^{1 / 2}(1-s)^{-1 / 2} d s}{s-x}=\int_{-1}^{1} \frac{s\left(1-s^{2}\right)^{-1 / 2} d s}{s-x}=-\int_{-1}^{1} \frac{(1+s)^{-1 / 2}(1-s)^{1 / 2} d s}{s-x}=\pi, \\
\frac{d}{d x} \int_{-1}^{1} \frac{F(s) d s}{1-x}=-\frac{F(1)}{1-x}-\frac{F(-1)}{1+x}+\int_{-1}^{1} \frac{F^{\prime}(s) d s}{s-x}, \\
\int_{-1}^{1} e^{\alpha t}\left(1-t^{2}\right)^{n-1 / 2} d t=\frac{\Gamma(n+1 / 2) I_{n}(-\alpha)}{\pi^{-1 / 2}(-1 / 2 \alpha)^{n}} \quad\left(I_{n}=\right.\text { modified Bessel function), } \\
\int_{-1}^{1} e^{\alpha t} \sin ^{-1} t d t=\frac{\pi}{\alpha}\left[\cosh \alpha-I_{0}(\alpha)\right], \\
\int_{-1}^{1} t^{n} e^{\alpha t} F(t) d t=\frac{d^{n}}{d \alpha^{n}} \int_{-1}^{1} e^{\alpha t} F(t) d t .
\end{gathered}
$$

6. Second example. Consider

$$
\int_{-1}^{1}[1+k(x-t)] \ln |x-t| f(t) d t=1
$$

where $k$ is a parameter. It is of interest to compare the solution of Eq. (12) with that obtained by setting $k=0$ and using Eq. (3); in particular, one may ask how large $k$ must be before its effect becomes important. The function $T(x)$ is found from Eq. (4) as

$$
T(x)=c_{0}[(-1+1 / 2 k)-k x]+c_{1}[(-1+k)-k x]+1 / 2 k c_{2}
$$

and two relations between these $c_{i}$ may be found by the method of Sec. 4:

$$
\begin{gathered}
c_{0}(\ln 2)+k c_{1}(1-\ln 2)=-1, \\
k c_{0}(1 / 2-\ln 2)-2 c_{1}+k c_{2}=0 .
\end{gathered}
$$

These two equations may be used to simplify the expression for $h(x)$, which becomes

$$
h(x)=\frac{\left(1-x^{2}\right)^{1 / 2}}{\pi}\left[c_{0}(-k \ln 2)\right]+\frac{1}{\pi}\left(\frac{\pi}{2}-\sin ^{-1} x\right)\left[c_{0}(1+k x)-k c_{1}\right] .
$$

Finally, Eq. (9) gives

$$
\begin{aligned}
f(x) & =\frac{d}{d x} \int_{x}^{1} \exp [k(t-x)] h^{\prime}(t) d t \\
& =-h^{\prime}(x)+k h(x)+k^{2} \int_{x}^{1} \exp [\alpha(t-x)] h(t) d t .
\end{aligned}
$$


Integrating Eq. (13) between -1 and +1 gives another relation between the $c_{i}$

$$
c_{0}(1-\ln 2) I_{1}(k)-c_{1} I_{0}(k)=0
$$

so that, solving for $c_{0}$ and $c_{1}$,

$$
c_{0}=-\frac{I_{0}(k)}{(\ln 2) I_{0}(k)+k I_{1}(k)}, \quad c_{1}=-\frac{(1-\ln 2) I_{1}(k)}{(\ln 2) I_{0}(k)+k I_{1}(k)}
$$

and the final solution may now be written down by substituting for $h(x)$ and the $c_{i}$ in Eq. (13). The special case of small $k$ is of interest; to the first order,

$$
f(x)=-\frac{1}{\pi \ln 2\left(1-x^{2}\right)^{1 / 2}}+k\left(1-\frac{1}{\ln 2}\right) \frac{x}{\pi\left(1-x^{2}\right)^{1 / 2}},
$$

where the first term is the well-known solution of Eq. (12) for $k=0$. The correction term bears a ratio to the first term of approximately $\left(\frac{1}{2} k x\right)$, which is of the expected magnitude.

To conclude, it may be remarked that to write out a formal solution is estimated to require about $2 n^{3 / 2}$ hours of labor for that case in which $P$ and $Q$ are $n$th order polynomials. Of course, once such a solution has been tabulated, it may be used for a number of kernels or parameters. Note also, as previously mentioned, the possibility of curvefitting. In particular, if a large number of terms are to be used, it may be worthwhile to set $P=1$ and represent the kernel behavior by $[1 n|x-t|+Q]$ alone; the $Q$ terms could then of course be absorbed directly into $g(x)$ and Eq. (3) used.

\section{REFERENCES}

1. N. Wiener and E. Hopf, Berlin Ber., Math. Phys. Klasse, 1931, p. 696

2. T. Carleman, Math. Z. 15, 111 (1922)

3. T. Carleman, Arkiv. for Mat., Astron., och Fysik 16, No. 26 (1922)

4. G. Latta, Tech. Rep. No. 32 of Appl. Math. and Stat. Lab., 1955, Stanford Univeraity, Calif.

5. N. Muskhelishvili, Singular integral equations, Transl. by J. R. M. Radok, Noordhof, Groningen, 1953

\section{PRIORITY ASSIGNMENT ON A WAITING LINE*}

\section{By S. A. DRESSIN" ${ }^{1}$ AND E. REICH ${ }^{2}$ (Santa Monica, California)}

Interest in queueing statistics when a priority system is involved is now widespread, especially in connection with communications applications. In this paper we consider a model described in the usual customer-counter terminology as follows.

Input. There are $r$ classes of customers, a particular class, labelled by the integer $p$, $(p=1,2, \ldots, r)$, being made up of customers of "priority" $p$. (Following an unfortunate, but standard, practice, priority $p_{1}$ will be said to be higher than priority $p_{2}$ if and only if $p_{1}<p_{2}$.) Customers of priority $p$ arrive as a Poisson process with interarrival-period distribution $\lambda_{p} e^{-\lambda_{p} x} d x, x>0$. Customers of different priorities arrive independently.

Queue-discipline. Once a customer's service has begun, it is permitted to proceed to completion. If the counter becomes empty, and there is at least one customer waiting,

*Received May 7, 1956.

${ }^{1}$ Major, U. S. M. C., U. S. Naval Postgraduate School, Monterey, Calif.

${ }^{2}$ Now at University of Minnesota. 